R Macastena-Maxhuni, V Grajqevci-Uka, B Abrashi, F Selimi. Hemato-Oncology Department, Pediatric Clinic, University Clinical Centre of Kosovo, Prishtina, Kosovo

Introduction Nephrobllastoma is a kidney cancer which usually appears in children and rarely in adults.

Aim The aim of the paper was the presentation of Tu Wilms cases in the Department of Hemato-Oncology at December 2008 till March 2012.

Materials and Methods In the paper are included 17 cases, 7 males $(41.17 \%)$ and 10 females $(58.82 \%)$, that are present in our department. They are of different sexes and ages. Meanwhile, regarding to tumor lateralization, 11 cases have been on the right kidney or $(64.70 \%), 5$ cases or $(29.41 \%)$ were located in the left kidney, and 1 case or $(5.88 \%)$ was on both kidneys. The examination is done based on hystory, physical examination, laboratory analysis, radiologic (X-ray, ECHO of abdomen and CT of torax and abdomen) and biopsy.

Results The preoperative chemotherapy is applied into 12 cases $(70.50 \%)$ and in 16 cases $(94.11 \%)$ is applied the treatment of pre and postoperative. According to SIOP 2001 protocol, one of them was accompanied abroad after the treatment. During the preoperative treatment the tumors mass is reduced from $25 \%-35 \%$. Two of these cases are in treatment for rellaps, one of them refused the operation after the preoperative treatment.

Conclusion Based on the data of this research, we conclude that the by diagnosing in the beginning of the stage, preoperative, a good surgical intervention and the postoperative treatment, has provided very good results and increases the longevity of these patients.

\section{THE ASSOCIATION OF ZINC DEFICIENCY WITH IRON DEFICIENCY}

doi:10.1136/archdischild-2012-302724.0753

B Arcagok, T Celkan, N Ozdemir, I Yildiz. Pediatric Hematology-Oncology Dept., Istanbul University, Cerrahpasa Medical Faculty, Istanbul, Turkey

Aim The aim of this study is to determine whether iron deficiency is associated with zinc deficiency.

Methods Ninety-eight patients with ID and/or IDA and 100 healthy controls who were examined at Cerrahpasa Medical Faculty, Pediatric and Child Health Department outpatient clinic were included prospectively in this study. Blood samples were collected from cases and controls for complete blood count, iron, TIBC, ferritin and zinc levels. The association between zinc levels and hematological and biochemical parameters were examined.

Results There was significant difference $(p<0.05)$ between zinc levels of the cases and controls, serum zinc levels were lower in cases compared to controls. Zinc deficiency was more frequent in children with iron deficiency and/or anemia (9.2\%) than those without iron deficiency (1\%). In cases, no significant difference was found ( $p>0.05)$ between zinc levels in relation to the absence or presence of anemia. There was no correlation between zinc levels and $\mathrm{Hb}, \mathrm{Htc}, \mathrm{MCV}$, ferritin or TIBC ( $p>0.05)$. However there was a positive correlation beween zinc levels and iron and TSI. In the control group, there was no statistical relation between zinc levels and $\mathrm{Hb}, \mathrm{Htc}, \mathrm{MCV}$, iron, TIBC, TSI and ferritin levels ( $p>0.05$ ).

Conclusion In our study, we detected significant lower zinc levels in children with iron deficiency before the onset of anemia. Therefore, we propose that the use of preparations containing both iron and zinc might be more effective in improvement of clinical signs of iron and zinc deficiency.
A Ahmadiafshar, A Ghadipasha, N Mousavinasab. Zanjan University of Medical Sciences, Zanjan, Iran

Background Several studies demonstrated some alterations in immune system of $\beta$-thalassemia major patients. The aim of this study was to assess the immunologic markers of these patients in comaprision with control group.

Method Immunologic markers including CD8, CD4 [T-lymphocyte], CD19 [B-lymphocyte], and CD56 [NK cell]) were assessed in thirty patients with $\beta$-thalassemia major (18 male and 12 female; under 18 years) and similar age and sex matched healthy controls. All patients had no infectious, malignant or chronic diseases. Complete blood count, and serum ferritine and iron also were measured. Statistical analysis performed by SPSS (v.15) software.

Results We did not found any abnormality in cellular and humoral system. However, mean CD56 level in thalassemia group were significantly lower than control group $(6.54 \pm 2.87 \%$ vs. $9.13 \pm 4.01 \%$; $\mathrm{p}=0.006$ ). Mean CD4 in thalassemia patients with spelenectomy was significantly lower than patients without splenectomy (31.8 $\pm 6.55 \%$ vs. $40.3 \pm 9.2 \%$; $=0.02$ ).

Conclusion NK cell marker in the patients with $\beta$-thalassemia major is lower than healthy individuals, that may be responsible for defects in innate immune system.

\section{DOES PARTIAL EXCHANGE TRANSFUSION PREVENT NEURODEVELOPMENTAL DISABILITY IN INFANTS WITH POLYCYTHEMIA?}

doi:10.1136/archdischild-2012-302724.0755

${ }^{1} \mathrm{H}$ Tatar Aksoy, ${ }^{1} \mathrm{R}$ ÖZdemir, ${ }^{2} \mathrm{Z}$ Eras, ${ }^{1} \mathrm{FE}$ Canpolat, ${ }^{3} \mathrm{U}$ Dilmen. ' $\mathrm{NICU}$, Zekai Tahir Burak Maternity and Teaching Hospital, Department of Neonatology; 'Zekai Tahir Burak Maternity and Teaching Hospital; ' ${ }^{3} Z e k a i$ Tahir Burak Maternity and Teaching Hospital/ Yıldırım Beyazıt University Department of Pediatrics, Ankara, Turkey

Partial exchange transfusion (PET) is traditionally suggested as treatment for neonates diagnosed with polycythemia. Nevertheless, late neurodevelopmental outcome of this treatment is controversial. We aimed to compare the neurodevelomental outcomes of the children who had history of neonatal polycythemia that treated with PET or not at 2 years old. Neonates who were hospitalized due to polycythemia between April 2009 and September 2009 included the study. Mental and psychomotor evaluations were performed using the Bayley Scales of Infant and Toddler Development Second Edition (BSID-II). The examiner was blinded to both group. 13 infants treated with PET and 21 not treated with PET were included the study. There were no statistically difference in respect to demographic and prenatal characteristics between groups. There were no statistically significant differences in immediate complication rates between groups, except indirect hyperbilirubinemia. Mental and psychomotor scores of the both groups were not statistically different. When the infants grouped into according to psychomotor scores $(>85$ and $\leq 85)$ more infants who were not treated with PET had psychomotor scores $\leq 85$ compared to the other group $(p=0.03)$. More infants who were not treated with PET had psychomotor scores between 70 and 84 . This means group who not treated with PET had more infants having mild psychomotor disability compared to other group. The main issue about the polycythemia treatment is late nerodevelopmental outcome. In our study, treatment with PET may protect the neonates with polycythemia from the late psychomotor disability.

\section{THE EFFECTIVENESS OF RDW-CV IN DIFFERENTIATING MICROCYTIC ANEMIA}

doi:10.1136/archdischild-2012-302724.0756

ML Neamtu, L Dobrota, MB Neamtu, FS Diac, II Simina. Pediatric Clinic Hospital Sibiu, Lucian Blaga University of Sibiu, Sibiu, Romania 